Sādhanā Vol. 40, Part 3, May 2015, pp. 925-943. (C) Indian Academy of Sciences

\title{
Stability of fluid flow through deformable tubes and channels: An overview
}

\author{
V SHANKAR
}

Department of Chemical Engineering, Indian Institute of Technology,

Kanpur 208016, India

e-mail: vshankar@iitk.ac.in

MS received 7 July 2014; revised 17 January 2015; accepted 6 February 2015

\begin{abstract}
The aim of this paper is to provide a systematic overview of the study of instabilities in flow past deformable solid surfaces, with particular emphasis on internal flows through tubes and channels. The subject is certainly more than five decades old, and arguably began with Kramer's pioneering experiments on drag reduction by compliant surfaces. This was immediately followed by the theoretical studies of Benjamin and Landhal in the early 1960s. Most earlier theoretical studies were focused on stability of external flows such as boundary layers, and used relatively simple wall models composed of spring-backed plates. There has been a resurgence in the field since the mid-1980s, and more attention was focused on internal flows through deformable tubes and channels. The wall deformation was described by both phenomenological spring-backed plate models and continuum linear viscoelastic solid models. All these studies predict several types of instabilities in flow past deformable surfaces. This paper will attempt to place the various theoretical results in perspective, and to classify the instabilities predicted by various studies. Recent studies have also emphasized the importance of using a frame-invariant constitutive model, such as the neo-Hookean model, for the solid deformation. Until recently, however, the field has been dominated by theoretical and numerical studies, with very little experimental observations to corroborate the theoretical predictions. Recent experiments in flow through deformable tubes and channels indeed show instability at Reynolds number much lower than their rigid counterparts, and the experimental observations are in qualitative agreement with some of the theoretical predictions. There have also been a few studies on the non-linear aspects of the instability using the weakly non-linear formulation to determine the nature of the bifurcation at the linear instability. A brief discussion on weakly nonlinear analyses is also provided in this paper.
\end{abstract}

Keywords. Hydrodynamic instability; flow through deformable tubes and channels; inviscid modes; wall modes; flow past deformable solid surfaces; laminar-turbulent transition. 


\section{Introduction}

There are many settings, both natural and technological, in which there is fluid flow past a deformable solid where the fluid stresses are strong enough to cause deformation in the solid, and the deformation of the solid in turn affects the nature of the fluid flow. For instance, in most biological flows, the fluid conveying vessel is made up of soft tissues, and hence is deformable (Grotberg \& Jensen 2004). Examples include blood flow in the circulatory system, and air flow in the respiratory system (Grotberg 2011). While the internal flow of blood and other fluids in biological systems has been well-studied ( $\mathrm{Ku} \mathrm{1997),} \mathrm{it} \mathrm{is} \mathrm{often} \mathrm{assumed} \mathrm{that} \mathrm{the} \mathrm{nature} \mathrm{of} \mathrm{the}$ flow would be qualitatively similar to flow in rigid conduits, and the focus is on the unsteady nature of the flow and secondary flows. There have been several studies (Pedley 2000; Grotberg $\&$ Jensen 2004) that focus on the collapsible nature of the flexible tube due to the pressure difference between the region outside the walls and the flow, and the dynamics of the flow inside the vessel in the highly collapsed state. However, the possible role of the deformable nature of the wall on the stability of the flow, in which there is not much change in the channel/tube cross-section, has not been addressed adequately in the bio-fluid dynamics literature. In addition, many applications in microfluidic devices use the soft elastomer polydimethyl siloxane (PDMS) as the material for fabrication (McDonald \& Whitesides 2002; Squires \& Quake 2005), and this opens up the possibility of exploiting the soft and deformable nature of the elastomeric walls to promote mixing in microfluidic applications. Since the Reynolds number for flows in such micron-scale devices is usually less than unity, flows are generally laminar. This places severe restriction on the transport rates in such devices, which are diffusion-controlled, and one potential way to improve mixing is to induce instabilities in these devices driven by the deformable nature of the wall. Thus, the understanding of the transition of laminar flow in tubes and channels with deformable walls is relevant to a wide variety of applications.

The study of stability of fluid flow past compliant walls has been an active area since 1960s, but the motivation for these earlier studies mostly stemmed from marine and aerospace applications, where the interest is in reducing the drag force (and hence the work done for propulsion) by the use of compliant coatings. This reduction could be achieved either by delay of the laminarturbulent transition or by the reduction of drag in the fully turbulent flow. Owing to the nature of the applications, the focus of these studies was largely on external flows, which usually involved the boundary layer flow past a compliant surface. However, the early experimental work of Lahav et al (1973) and Krindel \& Silberberg (1979) on gel-coated tubes suggested that the deformable nature of the wall could advance the transition in the gel-coated tube to Reynolds number much lower than the rigid-tube value of 2000. There was no follow-up of this early experimental work, and internal flows through deformable tubes and channels remained relatively unexplored until the late 1980s, when there was a resurgence in the subject.

The goal of this review paper is to provide a systematic overview of the subject of instability of internal laminar flows in deformable tubes and channels. The focus will primarily be on the models for the deformable wall, methods of analysis, results from various studies, and how best to unify the results into classes of instabilities. We will also make brief references to external flows since many of the instabilities studied are common to both internal and external shear flows. It is also appropriate here to refer to some earlier reviews on this subject: the reviews of Kumaran (2003), Heil \& Jensen (2003), Davies (2003), and Lucey \& Peake (2003) were part of the proceedings of the IUTAM symposium on this subject held in 2001. The present review will largely try to cover the developments since that symposium, but in the interests of completeness, and for the benefit of a general reader, we also provide brief discussions of all the relevant studies 
before 2001 as well. In addition, the review paper of Grotberg \& Jensen (2004) also provides a summary of flow through flexible tubes in the biological context.

The rest of the review is organized as follows: Section 2 provides a summary of the configurations considered and the models used for the fluid and solid. In section 3, we briefly describe the methods of analysis used for studying the stability of these flows. We then proceed to discuss the stability of flow at high Reynolds number in section 4 , where the inviscid instability mechanisms are discussed in detail. In section 5, we discuss a qualitatively new type of instability in the high- $R e$ regime called the "wall modes". In section 6 , we discuss the nature of stability at low Reynolds number. Section 7 provides a brief overview of weakly nonlinear stability of flow past deformable solid surfaces. Finally, section 8 provides the summary of the salient conclusions and suggestions for future work in this area.

\section{Systems and models}

The systems considered in this review (figure 1) involve canonical internal shear flows such as the plane Couette flow between a moving rigid plate and a deformable solid layer, plane Poiseuille flow in a deformable channel, and pressure-driven flow in a tube with deformable walls. In all these cases, while there is fluid flow past the deformable solid layer on one side, the deformable solid layer is assumed to be perfectly bonded to a rigid surface at the other side. This precludes phenomena like tube collapse due to pressure difference across the tube walls, as discussed in the earlier studies of Pedley (2000). Thus, the boundary conditions at the outer rigid surface are crucial for the kind of phenomena described in this review. In addition to the internal flow configurations shown in figure 1, we will also make references to the canonical external flow, viz., boundary layer flow past a compliant wall, in order to facilitate comparisons with earlier results in this area which were predominantly focused on boundary layer flows. In most of the earlier studies, the fluid was assumed to be Newtonian, and the same assumption will be made here as well. The governing equations for an incompressible Newtonian fluid are the Navier-Stokes mass and momentum conservation equations given by

$$
\nabla \cdot \mathbf{v}=0, \quad \rho\left[\partial_{t} \mathbf{v}+\mathbf{v} \cdot \nabla \mathbf{v}\right]=-\nabla p+\eta \nabla^{2} \mathbf{v} .
$$

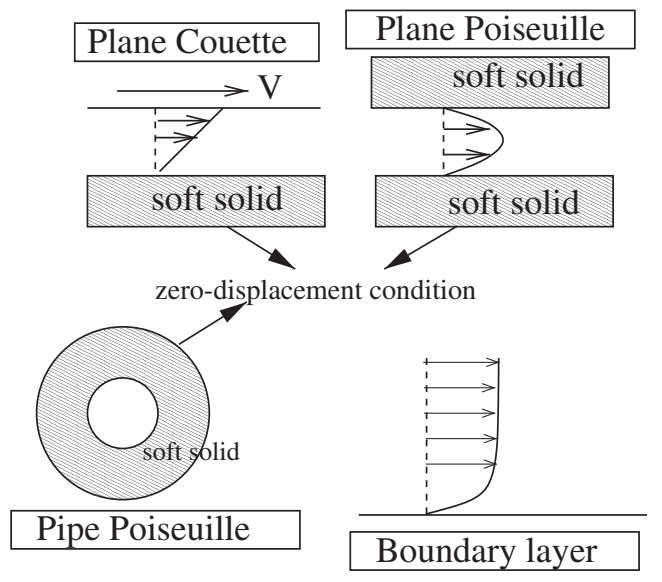

Figure 1. Schematic of the various internal flow geometries considered in this review. 
However, it must be mentioned here that there have been some studies (Shankar \& Kumar 2004; Kumar \& Shankar 2005; Choskshi \& Kumaran 2007; Neelamegam et al 2013), wherein the role of viscoleastic effects in the fluid on the stability has been analyzed.

\subsection{Continuum models for solid deformation}

The deformable solids considered in this review are typically soft elastomeric blocks in which a tiny rectangular slot/circular hole is carved out using a template. Such elastomeric solids of finite thickness are best modelled as a continuum elastic solid, while accounting for dissipative effects that are present in the polymer gel. One of the simplest models to describe deformation in the solid is the linearized elastic solid model (Landau \& Lifshitz 1989), modified to include dissipative viscous effects. Further, usually soft solids have bulk modulus to be much larger compared to their shear modulus, implying that the volumetric changes in deformation are negligible compared to shear deformations. Thus, most studies have assumed that the solid is incompressible. The deformation in the solid is described by a displacement field $\mathbf{u}(\mathbf{X})$, which represents the deviation of positions of material points from their reference values. The stress tensor for a linearized elastic solid is given by

$$
\sigma_{s}=-p_{s} \mathbf{I}+G\left(\nabla \mathbf{u}+\nabla \mathbf{u}^{T}\right),
$$

where $p_{s}$ is the pressure-like variable in the solid that enforces the incompressibility condition in the solid, $G$ is the shear modulus of the solid material. The dissipative effects in the solid can be incorporated in a simple manner as

$$
\sigma_{s}=-p_{s} \mathbf{I}+G\left(\nabla \mathbf{u}+\nabla \mathbf{u}^{T}\right)+\eta_{s} \frac{\partial}{\partial t}\left(\nabla \mathbf{u}+\nabla \mathbf{u}^{T}\right),
$$

where $\eta_{s}$ is the viscosity of the solid. It is assumed that, in the interests of simplicity, the viscosity is a frequency-independent constant. The governing mass and Cauchy momentum equations for an incompressible linearized elastic solid are

$$
\begin{aligned}
\nabla \cdot \mathbf{u} & =0, \\
\rho_{s}\left(\frac{\partial^{2} \mathbf{u}}{\partial t^{2}}\right) & =\nabla \cdot \boldsymbol{\sigma}_{s} .
\end{aligned}
$$

These equations must be supplemented with boundary conditions at the rigid surface where the elastic material is assumed to be perfectly bonded, and with kinematic and stress continuity conditions at the interface between the fluid and solid. While the linear elastic solid model is simple to implement, it has a fundamental deficiency in that it is applicable only if the strain in the solid is $\ll 1$. This arises because of the fact that the nonlinear term in the strain measure has been neglected for small strains, and this neglect renders the linearized elastic model to violate principle of material-frame indifference. It might be anticipated that in a linear stability analysis the nonlinear strain measure would not play a role, since nonlinear terms are neglected by definition in a linear analysis. However, this is incorrect, since the linearization is carried out about a deformed base state, and if the base state deformation is not small, then non-trivial terms would arise in the stability analysis coupling the base-state deformation and fluctuations in the deformation field. The lacunae involved in using a linearized elastic model were first pointed out by Gkanis \& Kumar (2003) in the context of plane Couette flow past a deformable solid layer. Much of the earlier work in this subject were based on the linearized elastic model 
(Yeo \& Dowling 1987; Yeo 1988; Yeo et al 1994; Lucey \& Peake 2003; Kumaran 1995, 1998a, b; Shankar \& Kumaran 1999, 2000), and it is important to examine whether the predictions obtained using the linearized elastic model would be modified by using a more accurate nonlinear model. We will specifically address this issue at various points in this review. One of the simplest models that use a frame-invariant, non-linear strain measure is the neo-Hookean model for a solid material (Malvern 1969; Holzapfel 2000).

The governing equations for the fluid (Eq. 1) are written, as is customary, in terms of spatial (Eulerian) coordinates $(\mathbf{x}=x, y, z)$. It is possible to express the dynamical quantities and governing equations in the solid also in a consistent Eulerian fashion (Chokshi 2007; Choskshi \& Kumaran 2008). Equivalently (following Gkanis \& Kumar 2003), it is convenient to refer the governing equations for the solid in terms of a reference (Lagrangian) configuration, where the independent variables are the positions $\mathbf{X}=(X, Y, Z)$ of material particles in the reference (i.e. unstressed solid) configuration. Chokshi (2007) has shown that the Eulerian-Eulerian and Eulerian-Lagrangian approaches are equivalent, and yield the same result for the eigenvalues. In a recent work, Ma \& Ng (2009) have used an entirely Lagrangian approach for both the fluid and solid to analyse the propagation of waves in an elastic tube, when there is no base flow in the fluid. However, their approach is not easily applicable to the case when there is a base flow in the fluid, as is the case in the class of problems considered in this review.

The spatial $(x, y, z)$ coordinate system used here for fluid motion is identical to the reference coordinate system in the unstressed configuration $(X, Y, Z)$ for the deformable solid. In the deformed state of the solid, the current position of a material particle is denoted in the Lagrangian description by $\boldsymbol{w}(\mathbf{X})$. The deformable solid is modelled as an incompressible neo-Hookean viscoelastic solid and the mass and momentum conservation equations governing the dynamics of solid are given as (Malvern 1969; Holzapfel 2000):

$$
\begin{aligned}
\operatorname{det}(\mathbf{F}) & =1, \\
\rho_{s}\left[\frac{\partial^{2} \boldsymbol{w}}{\partial t^{2}}\right]_{\mathbf{X}} & =\nabla_{\mathbf{X}} \cdot \mathbf{P} .
\end{aligned}
$$

In the above equations, $\mathbf{F}$ is the deformation gradient tensor defined as $\mathbf{F}=\nabla_{\mathbf{X}} \boldsymbol{w}$ and $\mathbf{P}$ is the first Piola-Kirchhoff stress tensor. The first Piola-Kirchhoff stress tensor is related to Cauchy stress tensor by $\mathbf{P}=\mathbf{F}^{-1} \cdot \boldsymbol{\sigma}$. The Cauchy stress tensor for the neo-Hookean viscoelastic solid is split into an elastic part, $\sigma_{\mathbf{e}}$, and a dissipative part, $\sigma_{d}$ (Ma \& Ng 2009; Destarde \& Saccomandi 2004; Beatty \& Zhou 1991; Fosdick \& Yu 1996):

$$
\begin{gathered}
\sigma=\sigma^{e}+\sigma^{d} \\
\sigma^{e}=-p_{s} \mathbf{I}+G \mathbf{F} \cdot \mathbf{F}^{T} ; \quad \sigma^{d}=\eta_{s} \frac{\mathrm{d}}{\mathrm{d} t}\left(\mathbf{F} \cdot \mathbf{F}^{T}\right) .
\end{gathered}
$$

Here $G$ is the shear modulus of the solid material, and $\eta_{s}$ is the (frequency-independent) viscosity of the solid. To simplify the stability analysis, we assume a frequency-independent viscosity to describe dissipative effects in the solid medium. The neutral stability curves obtained from this assumption can be extended to a solid with frequency-dependent viscosity by following an iterative procedure described in Muralikrishnan \& Kumaran (2002). A simple single-mode Maxwellian model for the viscoelastic response of the solid has been used in Chokshi \& Kumaran (2008). In the above governing equations for a solid layer, the density of the solid is assumed to be equal to the fluid density because the densities of commonly used polymeric 
materials are usually not very different from those of various liquids. Thus, the ratio of solid to fluid density $\rho_{s} / \rho$ will be close to one and for small differences in densities we expect that the qualitative prediction of the present study will remain unchanged. Further, if there is a pressure gradient in the fluid that is dependent on the flow direction, then the base-state deformation in the solid will also be a function of the flow direction. This would mean that the problem can be treated only in a "local" manner in the classical normal mode analysis. In principle, one must then resort to a more rigorous spatio-temporal analysis for such configurations.

\subsection{Spring-backed plate model}

Here we briefly describe the spring-backed plate model that has been widely used for boundary layer flows (Carpenter \& Garrad 1985, 1986; Carpenter \& Gajjar 1990; Gajjar \& Sibanda 1996), as well as in channel flows (Davies \& Carpenter 1997; LaRose \& Grotberg 1997). The system consists of a fluid of thickness $R$ which is bounded at $z=R$ by a rigid surface moving at a constant velocity $V$, as shown in figure 2 . At the lower boundary $z=0$, there is an infinitesimal plate which is connected to a rigid base by springs. In this spring-backed plate model (LaRose \& Grotberg 1997; Carpenter \& Morris 1990), the flexible wall is modelled as a spring-backed plate which can deform both in the horizontal and vertical directions. The displacement in the horizontal and vertical directions, denoted respectively by $u_{x}$ and $u_{z}$, represent the deviation of the material points in the wall material from their equilibrium positions. In the previous studies, a constitutive equation of the following type

$$
\left(M \partial_{t}^{2}+D_{n} \partial_{t}+B \partial_{x}{ }^{4}-T \partial_{x}{ }^{2}+K\right) u_{z}=n_{i} \tau_{i j} n_{j},
$$

has been used for the normal displacement of the membrane $\left(u_{z}\right)$. Here $\tau_{i j}$ is the total stress tensor in the fluid and $n_{i}$ is the unit normal to the flexible surface. In most previous studies, the tangential displacement $\left(u_{x}\right)$ is set equal to zero, presumably on the basis that at high Reynolds number, the tangential velocity and stress conditions do not play an important role. However, this expectation proves to be incorrect, as will be explained later in this review. Some studies (LaRose \& Grotberg 1997; Carpenter \& Morris 1990) augment the above condition by another relation between the tangential stress and the tangential displacement:

$$
\left(M \partial_{t}^{2}+D_{t} \partial_{t}-E \partial_{x}^{2}\right) u_{x}=t_{i} \tau_{i j} n_{j}
$$

In the above Eqs. (10) and (11), $n_{i} \tau_{i j} n_{j}$ and $t_{i} \tau_{i j} n_{j}$ are respectively the normal and tangential fluid stress at the interface, and $t_{j}$ is the unit vector tangential to the flexible surface. $D_{n}$ is the

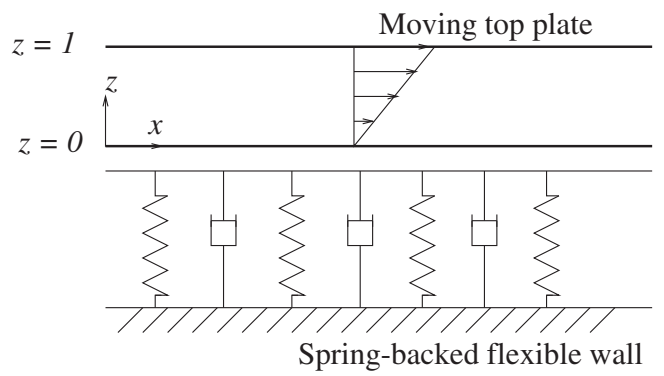

Figure 2. Schematic diagram of spring-backed compliant walls. 
normal wall damping coefficient, $D_{t}$ is the tangential wall damping coefficient, $B$ is the bending stiffness of the plate, $T$ is the longitudinal tension per unit width, $K$ is the spring stiffness of the membrane, $E$ is the product of elastic modulus and plate thickness, $M$ is the mass per unit area of the membrane, $x$ is the Cartesian coordinate along the wall, $z$ is the direction normal to the wall, $\partial_{x} \equiv \partial / \partial x, t$ is the dimensional time variable and $\partial_{t} \equiv \partial / \partial_{t}$.

\subsection{Linearization of continuity conditions at the fluid-solid interface}

In this section, we provide the details of the linearization of the interfacial continuity conditions at the fluid-solid interface leading to the linearised conditions . In this review, an Eulerian framework is used to describe the fluid dynamics, while a Lagrangian approach is used for the deformation in the solid. The basis underlying the derivation is the fact that a material particle's Lagrangian label remains unchanged upon introduction of perturbations to the fluid-solid interface. If a material point on the unperturbed interface (figure 3$)$ is located at $(X, Z=0)$ in the unstressed configuration, its label remains the same even in the perturbed interface. As shown in figure 3, a material particle $P_{0}$ with Lagrangian label $(X, Z=0)$ in the unstressed state is displaced to point $P\left(\bar{w}_{X}, 0\right)$ in the deformed base state. Upon introduction of perturbations to the interface, the point $P$ moves to $P^{\prime}\left(\bar{w}_{X}+w_{X}^{\prime}, w_{Z}^{\prime}\right)$, where, $w_{X}^{\prime}$ and $w_{Z}^{\prime}$ are the Lagrangian displacements of the material particle $P$ from the deformed base-state of the solid. The conditions at the perturbed fluid-solid interface $(X, Z=0)$ are continuity of velocities and stresses. Denoting $F$ and $S$ to be the fluid and solid dynamical quantities, respectively, the continuity conditions at the perturbed interface can be schematically written as

$$
\left.F(x, z, t)\right|_{P^{\prime}}=\left.S(X, Z, t)\right|_{P^{\prime}}
$$

The current position of the point $P^{\prime}$ is unknown a priori, and since Eulerian coordinates are used for the liquid layer, any fluid dynamical quantity on the left side of Eq. (12) is expressed by Taylor expansion about the known position of the unperturbed flat interface $(z=0)$. Thus, the left side of Eq. (12) is given as

$$
\left.F(x, z, t)\right|_{P^{\prime}}=\left.F\right|_{P(z=0)}+h(x)\left(\frac{\partial F}{\partial z}\right)_{P(z=0)},
$$

where $h(x)=w_{Z}^{\prime}(X, Z=0)$ is the vertical displacement of the material particle $P_{0}$ at its current position $P$. The current coordinates of the material particle $P_{0}$ at the point $P^{\prime}$ can also be expressed in terms of the displacements as $x=\bar{w}_{X}(X, Z=0)+w_{X}^{\prime}(X, Z=0)$. Defining the displacement in the base state about the unstressed state as $\bar{u}_{X}(Z)$, the $x$-coordinate of material point $P_{0}$ can be written as $x=X+\bar{u}_{X}(Z=0)+w_{X}^{\prime}(X, Z=0)$. For a specified current position $x$, the Lagrangian coordinate of the material point $P_{0}$ is obtained from the fundamental definition $X=x-\bar{u}_{X}(Z=0)-w_{X}^{\prime}(X, Z=0)$. When the solid dynamical quantities are

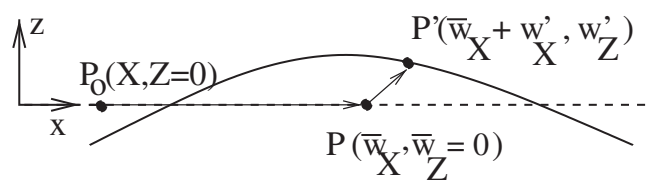

Figure 3. Schematic diagram illustrating the application of boundary conditions over the perturbed fluidsolid interface. 
evaluated at this Lagrangian coordinate, this would be tantamount to evaluating the quantities at the perturbed interface. Therefore, the right side of Eq. (12) is simply given as

$$
\left.S(X(x), Z, t)\right|_{P^{\prime}}=S\left(X=x-\bar{u}_{X}(Z=0)-w_{X}^{\prime}(X, Z=0), Z=0, t\right) .
$$

In other words, a Taylor-expansion (in the vertical Lagrangian coordinate) about the unperturbed interface for the solid is not required, as the perturbed interface is in fact described by $X(x), Z=$ 0 in the Lagrangian description of the solid. If an Eulerian framework is used (Chokshi 2007), the expansion about $z=0$ is justified for the solid.

Following the above procedure, the continuity of normal velocity at perturbed fluid-solid interface is given exactly by

$$
\left.v_{z}(x, z)\right|_{P^{\prime}}=\left(\frac{\partial w_{Z}}{\partial t}\right)_{X=x-\bar{u}_{X}(Z=0)-w_{X}^{\prime}(X, Z=0), Z=0} .
$$

Using a Taylor expansion to express left side of Eq. (15) and retaining the terms that are linear in perturbation quantities, Eq. (15) can be written as

$$
v_{z}^{\prime}(x, z=0)=\left(\frac{\partial w_{Z}^{\prime}}{\partial t}\right)_{X=x-\bar{u}_{X}(Z=0)-w_{X}^{\prime}(X, Z=0), Z=0} .
$$

Expressing the perturbation variables in terms of Fourier modes, i.e., on substituting $v_{z}^{\prime}(x, z=$ $0)=\tilde{v}_{z}(z=0) \exp [\mathrm{i} k(x-c t)]$ and $w_{Z}^{\prime}=\tilde{w}_{Z}(Z=0) \exp [\mathrm{i} k(X-c t)]$ and rearranging, we obtain

$$
\tilde{v}_{z}(z=0) \mathscr{E}=-\mathrm{i} k c \tilde{w}_{Z}(Z=0) .
$$

where $\mathscr{E} \equiv \exp \left[\mathrm{i} k\left(\bar{u}_{X}(Z=0)+w_{X}^{\prime}(X, Z=0)\right)\right]$. The remaining three interfacial conditions (tangential velocity and the normal and tangential stress continuity conditions) can be similarly derived. For example, the linearised tangential velocity balance is

$$
\tilde{v}_{x}(z=0) \mathscr{E}+\left.\tilde{w}_{Z}(Z=0) \mathrm{d}_{z} \bar{v}_{x}\right|_{z=0}=-\mathrm{i} k c \tilde{w}_{X}(Z=0) .
$$

The factor $\mathscr{E}$ multiplies fluid perturbation variables in all the four interfacial conditions, and hence must be linearised within a linear stability analysis. This can be achieved by noting that $w_{X}^{\prime} \ll \bar{u}_{X}$, thus yielding within a linear analysis $\mathscr{E} \approx \mathscr{E}_{1}=\exp \left[\mathrm{i} k\left(\bar{u}_{X}(Z=0)\right]\right.$. The base-state displacement $\bar{u}_{X}(Z=0)$ of the wall at the unperturbed interface is independent of $X$, and hence is a constant for a fixed value of $\Gamma$. All the four interfacial conditions have $\mathscr{E} 1$ multiplying the fluid quantities, and the differential equations governing the fluid are linear and homogeneous. The perturbation fluid velocities and pressure in the governing equations can then be multiplied by the quantity $\mathscr{E} 1$, and that will leave the Orr-Sommerfeld equation for the fluid invariant. Thus, the presence of a constant scaling factor $\mathscr{E} 1$ multiplying the fluid dynamical quantities in the linearised interface conditions does not alter the eigenvalue $c$.

In addition, the neo-Hookean solid exhibits a non-zero first normal stress difference under shear deformation (Macosko 1994), and it is well-known in viscoelastic fluid flows (Renardy 1988) that a jump in the first-normal stress difference across a two-fluid interface could lead to an instability. Mathematically, this is due to the fact that when the tangential stress continuity condition is implemented, terms of the type $\mathbf{t} \cdot \boldsymbol{\sigma} \cdot \mathbf{n}$ appear, and when the interface is perturbed, the projection of the stress along the perturbed normal yields a linear term proportional to the jump in the first-normal stress difference (Chen 1991). In a similar manner, even for Newtonian 
fluid flow past a neo-Hookean solid medium, there is a jump in the first normal stress difference across the fluid-solid interface, since the first normal stress difference is absent in the Newtonian fluid. Gkanis \& Kumar (2003) first pointed out that this yields a short-wave instability much similar to that in the interface between two viscoelastic fluids.

\section{Methods of analysis}

The traditional approach (Chandrasekhar 1981; Drazin \& Reid 1981) to study hydrodynamic instabilities is to impose infinitesimal perturbations on the base-state, and linearize the governing equations about the base-state since the perturbations are deemed to be small. In flow past deformable surfaces, this would mean that the governing equations of both the fluid and solid are linearized about their respective base states. In addition, the interface between the fluid and solid is not known a priori, and hence it is necessary to linearize the boundary conditions about the unperturbed interface. This gives rise to new couplings between base-state quantities and the perturbations. Once the governing equations and interface conditions are linearized, this yields a coupled problem in which the fluid governing equations have a first-order time derivative, while the solid governing equations have a second-order time derivative. For translationally invariant flows (say, in the $x$-direction), Fourier transforms could be used to express the $x$-dependence in terms of Fourier modes with a continuously varying wavenumber $k$. Thus, a two-dimensional disturbance $f(x, y, t)$ could be expected to be written as

$$
f(x, y, t)=\tilde{f}(y, t) \exp [\mathrm{i} k x] .
$$

In the traditional approach, the time-dependence of the disturbances is assumed to be exponential, and one writes

$$
\tilde{f}(y, t)=\tilde{\phi} \exp [s t] .
$$

Here, $s$ is the growth rate of perturbations. In temporal stability, $s$ is a complex quantity, with its real part signifying whether the flow is unstable $(\operatorname{Re}[s]>0)$ or stable $(\operatorname{Re}[s]<0)$. Here, the wavenumber $k$ is stipulated to be a real quantity. In contrast, in spatial stability, the quantity $s$ is purely imaginary, with $s=\mathrm{i} \omega$, and the wavenumber $k$ is a complex quantity. When the flow is neutrally stable (i.e. no growth or decay of perturbations, either temporally or spatially), then both temporal and spatial modes coincide. In this review paper, we will restrict ourselves to temporal modes. For a given wavenumber $k$, there could be many eigenvalues $s$ (often referred to as the eigenspectrum or simply the spectrum), and as $k$ is varied continuously, if there is at least one eigenvalue (or, "mode") with $\operatorname{Re}[s]>0$, then the flow is deemed to be unstable, since there is exponential growth of perturbations. If for a given set of physical parameters (e.g. Reynolds number), there is no mode with $\operatorname{Re}[s]>0$, then the flow is deemed to be stable, since all perturbations would decay asymptotically as $t \rightarrow \infty$. This methodology is often referred to as the "normal mode" analysis in the literature.

However, as has been pointed out since the early 1990s (Schmid \& Henningson 2001; Schmid 2007), asymptotic decay of all modes as $t \rightarrow \infty$ does not necessarily mean that the disturbances decay at all times. Indeed, there have been many studies for flow through rigid channels and tubes, which argue that this "transient" growth could be relevant to the experimentally observed laminar-turbulent transition. This is because of the glaring discrepancy between theoretical predictions (based on asymptotic growth/decay of perturbations) and experimental observations in rigid conduits. For instance for fully developed flow in a pipe, the normal mode analysis predicts that the flow is stable at all Reynolds number, but it is known experimentally that the flow 
becomes unstable at $R e \sim 2000$. It has been suggested (Schmid 2007) that the non-normal nature of the underlying differential equation and its associated non-orthogonal eigenfunctions lead to this transient growth. Whether such transient growth phenomena are relevant to flow past deformable surfaces is a matter that can only be settled by comparing the predictions from the traditional normal mode analysis with experimental results. This is the viewpoint that will be taken in this review paper, viz., to restrict ourselves to normal mode analysis, and evaluate its predictions vis-a-vis experiments.

\section{High Reynolds number inviscid modes}

In the limit of high Reynolds number, viscous effects are usually negligible compared to inertial effects in the fluid, except over thin regions near the wall, where there is a viscous "wall layer" required to enforce the tangential velocity and stress conditions. In order for the elastic stresses in the deformable wall to be important in the coupled fluid-solid problem, it is required that the elastic stresses should be of the same order as inertial stresses in the fluid, i.e. $\rho V^{2} \sim G$. We refer to these class of modes as inviscid modes. The early literature on this subject has predominantly focused on the high- $R e$ limit, presumably because of the focus on marine and aerospace applications, and delay in transition. The earlier works of Benjamin $(1960,1963)$ and Landahl (1962) exclusively focused on this limit, and modelled the wall compliance using a (possibly complex) admittance of the wall, which relates the normal wall displacement to the fluid pressure. One of the issues that gained attention in the earlier studies is the role of wall compliance on the Tollmien-Schlichting (TS) instability mode present in rigid surfaces. Benjamin (1960) extended the classical analysis of the Tollmien-Schlichting instability to show that a non-dissipative compliant wall tends to stabilize the TS mode. On the other hand, damping in the wall had a destabilizing role on the TS mode. Benjamin (1963) proposed a three-fold classification of instabilities in flow past compliant surfaces. The TS mode is referred to as class A type wave in Benjamin's classification because of the destabilizing effect of wall damping on the instability. In addition to the TS mode, the compliance of the wall can by itself trigger new instabilities absent in rigid surfaces. The compliance-induced instabilities were classified as Class B or C based on whether modal coalescence is absent or present. Later, Carpenter $\&$ Garrad $(1985,1986)$ referred to the unstable modes induced by wall compliance as "flowinduced surface instabilities" (FISI), in which they distinguished two types of modes. One type of instability is referred to as the "travelling wave flutter", which requires the presence of the critical layer (Drazin \& Reid 1981) in which the wavespeed of the disturbance is equal to the local base flow velocity. One of the linearly independent solutions to the inviscid stability equation has a logarithmic singularity at the critical point, and the correct branch of the logarithm is chosen by matching the inviscid solution with the full viscous solution. This induces a phase shift between the fluid pressure and the normal wall displacement, leading to a net work done by the fluid at the wall. This has also been shown to occur in channel flows by Davies \& Carpenter (1997). The presence of damping in the wall leads to a stabilizing effect on this instability. Because of this reason, the travelling wave flutter (TWF) mode is classified as class B waves in Benjamin's classification (figure 4).

In addition to the TWF mode, previous studies also predict a "static divergence" (SD) instability (absent in flow past rigid surfaces), in which wall damping plays a destabilizing role. This instability occurs when the phase speed of the disturbances vanish at the point of instability, and the instability mode appears as a standing wave (and hence called "static" divergence). First, it can be modelled using potential flow (Lucey \& Carpenter 1992), and therefore flow shear only 


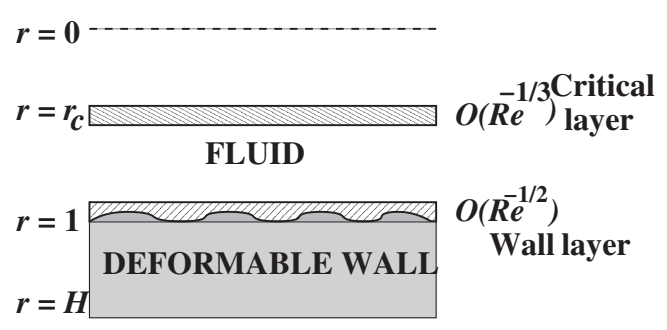

Figure 4. Schematic diagram showing the flow structure for inviscid modes.

plays a secondary role in the determination of its onset flow speed. When a compliant wall of infinite extent is modelled, wall damping is required to precipitate divergence and this has led it to be described as Class A instability; Landahl (1962) explained why this phenomenon occurred in terms of the imbalance of fluid and structural forces caused by the introduction of damping. The mechanism behind this instability is explained as follows. When the structural forces of the compliant wall are exceeded by the pressure forces exerted by the fluid, then the instability that arises is called a divergence instability (Davies 2003), and wall damping facilitates this imbalance of forces. The divergence instability mechanism can also be triggered by other means, such as when there is flow past a flexible solid surface with finite-lengths, where there is an interplay of end effects instead of wall damping. The equivalence of damping effects on an infinite wall and finite-length effects was shown by Lucey \& Carpenter (1993). Accordingly, divergence is best categorised as a Class $\mathrm{C}$ instability since the critical values for its onset are insensitive to damping. Crucially, the divergence instability does not require the presence of the critical layer (where the phase speed of disturbances equals the local base fluid velocity) unlike the TWF mode. Indeed, the effect of critical layer is to stabilize the divergence instability (Davies 2003). Owing to the role of damping, the divergence instability comes under the category of Class A waves in Benjamin's classification. At high $R e$, in addition to the critical layer where viscous effects are important, there is also a thin region close to the compliant wall where viscous effects become important in order to enforce the tangential velocity and stress continuity conditions. Incorporating this wall layer also seems to have opposing roles on the TWF and SD modes: while SD is destabilized by the wall layer, TWF mode is stabilized. Thus, while TS and SD modes are classified as class A, TWF is classified as class B in Benjamin's classification. Under certain parametric regimes, it was shown that TS (class A) and TWF (class B) modes coalesce to form a Kelvin-Helmholtz type instability, and this is referred to as class C waves (Sen \& Arora 1987) (figure 5).

In the limit of high-Re, it is possible to generalize the classical theorems of hydrodynamic stability for determining stability in the inviscid limit (Drazin \& Reid 1981). Yeo \& Dowling (1987) generalized these theorems for planar flows such as boundary layers, and showed that the equivalent of Rayleigh's inflexion point theorem to flow past flexible surfaces takes the following form: Inviscid perturbations to mean flow in a 2-D flexible channel is unstable only if $U U^{\prime \prime}<0$ somewhere in the flow domain. The normal deformation of the wall changes the Rayleigh's inflexion point theorem for rigid channels, which says that for flow in a rigid channel is unstable only if $U^{\prime \prime}=0$ somewhere in the flow. Thus, for the flow to be unstable in a flexible channel, there is no need to have an inflexion point, and it is sufficient if $U^{\prime \prime}$ is negative somewhere in the flow. This is true for fully developed flow in a flexible channel, and hence the flow could become unstable in the inviscid limit. This inviscid instability was analyzed by 


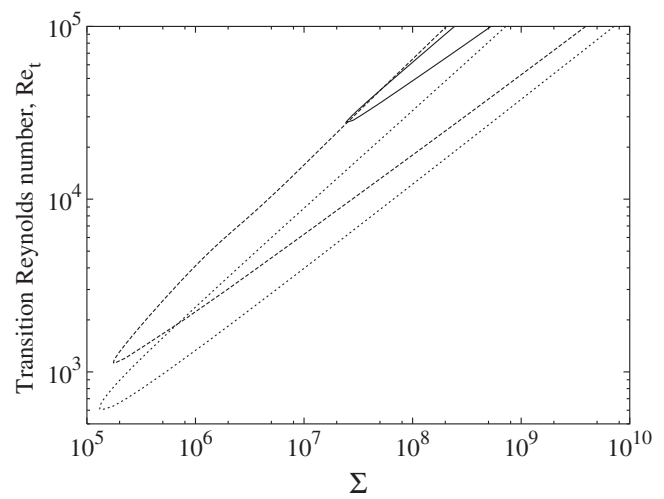

Figure 5. Neutral stability curves in the $R e-\Sigma$ plane for stability of fully developed flow to nonaxisymmetric disturbances. Results (from Shankar \& Kumaran (2000)) shown for $H=2, n=1, k=3$, and $\eta_{r}=0$.

Davies \& Carpenter (1997) for flow in a compliant channel. The equivalents of classical theorems of inviscid instability for the axisymmetric case of flow in a tube were derived by Kumaran (1996) and Shankar \& Kumaran (2000). This showed that fully developed flow in a deformable tube could be unstable in the inviscid limit to non-axisymmetric disturbances, while the flow in the developing region as well as flow in a (slowly) converging tube could become unstable in the inviscid limit to axisymmetric disturbances.

While the classical theorems are suggestive of inviscid instabilities in a deformable tube, they are merely necessary conditions, and not sufficient conditions. However, these theorems are useful in identifying velocity profiles that are stable in the inviscid limit. It is therefore necessary to carry out the stability analysis in order to examine the stability of the flows in the inviscid limit. This was carried out by Shankar \& Kumaran $(1999,2000)$ respectively for non-parabolic flows to axisymmetric disturbances, and for parabolic flow to non-axisymmetric disturbances. First, an inviscid analysis is carried out where viscous effects are neglected in the fluid. In this case, when the values of $c_{r}$ are such that it is in the range $0 \leq c_{r} \leq U_{\text {max }}$, then there is a critical point at which the one of the solutions to the inviscid stability equation has a logarithmic singularity. By choosing the correct branch of the logarithm (by appealing to the full viscous equations and by carrying out a matched asymptotic expansion near the critical point), it is possible to solve the problem in the inviscid limit, and these studies show that the flow is indeed unstable in the inviscid limit. In the inviscid limit, the tangential velocity and stress conditions cannot be applied, and hence this instability does not depend on whether the deformable wall allows for tangential wall motion or not. Since $\rho V^{2} \sim G$ in this limit, we can represent this relation as $\operatorname{Re} \propto \Sigma^{1 / 2}$ where $R e$ is the Reynolds number for flow, and $\Sigma=\rho G R^{2} / \eta^{2}$ is the non-dimensional wall elasticity. This is the expected characteristic scaling for inviscid flow, and the full numerical calculation shows that at sufficiently high $R e$, this scaling is observed for inviscid modes. Interestingly, numerical calculations show that this instability continues to very low $R e \sim 100$ for nonaxisymmetric disturbances in a deformable tube.

When comparing the analysis and results of Shankar \& Kumaran $(1999,2000)$ with earlier results in boundary layer flows and compliant channel flows, it appears that what Shankar \& Kumaran $(1999,2000)$ identify as "inviscid modes" are very similar to the TWF modes of Davies \& Carpenter (1997), since both involve the presence of the critical layer for the instability in the inviscid limit. As for the SD mode in a deformable tube made of elastic continuum, the 


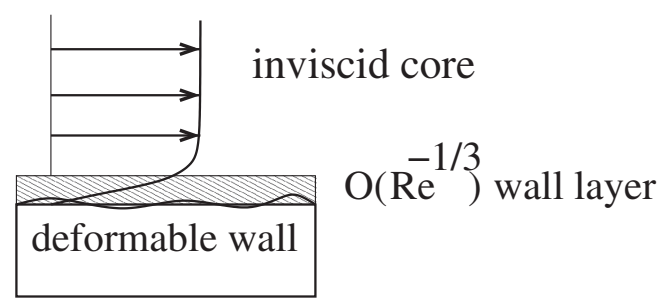

Figure 6. Schematic diagram showing the flow structure for wall modes.

dissipative term in the solid is proportional to $k c \eta_{r} / R e$. For inviscid modes, $c \sim O(1)$ and $R e \gg 1$, and for $\eta_{r} \sim O(1)$, this term becomes subdominant in a deformable tube, unless $\eta_{r}$ itself becomes unphysically large of $O\left(R e^{-1}\right)$. If we discount this unrealistic situation, it is safe to conclude that the SD modes found in compliant walls Davies \& Carpenter (1997) will not be present in deformable tubes made of finite viscoelastic continua (figure 6).

\section{High Reynolds number wall modes}

There is another class of modes at high $R e$, in which the wavespeeds $c$ are such that $c / V_{\max } \sim$ $R e^{-1 / 3}$, and in this limit, the viscous effects are confined to a thin region ("wall layer") of thickness $R e^{-1 / 3} R$ close to the wall. Here, $R$ is the radius of the tube, and $V_{\text {max }}$ is the maximum velocity of the base flow. It has been shown (Shankar \& Kumaran 2001a) that in this limit, the viscous stresses in the wall layer balance the elastic stresses in the solid, and the inviscid outer flow in the bulk of the fluid is irrelevant to this instability. At a scaling level, the viscous stresses in the wall layer would scale as $V_{\max } \eta /\left(R R e^{-1 / 3}\right)$ and the elastic stresses in the deformable wall would scale as $G$ (its shear modulus), and by balancing these two, one obtains $\operatorname{Re} \propto \Sigma^{3 / 4}$ for unstable wall modes. This is qualitatively different from inviscid modes, which scale as $R e \propto$ $\Sigma^{1 / 2}$. In order to capture the wall modes, it is appropriate to carry out an asymptotic analysis with the small parameter $R e^{-1 / 3}$ in the limit $R e \gg 1$. The (eigenvalue) wavespeed $c$ is expanded in an asymptotic series $c=c^{(0)}+R e^{-1 / 3} c^{(1)} \ldots$. The analysis indicates that to leading order, the linearized equations in the deformable wall get decoupled from the fluid, and the interface conditions degenerate to zero normal displacement and zero tangential stress conditions. The leading wavespeed $c^{(0)}$ is thus determined is purely real, and admits many solutions. These represent the free shear waves (of $O(G / \rho)^{1 / 2}$ ) of the solid layer. However, the viscous wall layer appears in the calculation of the first correction $c^{(1)}$, and it turns out that when $\Gamma=V_{\max } \eta /(G R)$ exceeds a critical value, the flow becomes unstable. There are multiple unstable modes at the first correction, corresponding to the multiple (real) wavespeeds at the leading order. Results from the asymptotic analysis are in excellent agreement with numerical solutions (see Shankar \& Kumaran 2001a, 2002). Another important feature that emerges out of the asymptotic analysis is that the instability crucially depends on the presence of tangential wall motion in the deformable solid. While it is naturally present for viscoelastic continua, many prior studies have used springbacked membranes that undergo only normal motion. It was shown (Shankar \& Kumaran 2002) that in such cases, the wall mode instability ceases to exist. Since the wall mode instability is dominated by the wall layer in the fluid, it is expected to be a generic feature in high- $R e$ shear flows past a deformable surface, as long as there is a finite velocity gradient at the wall (figure 7). 


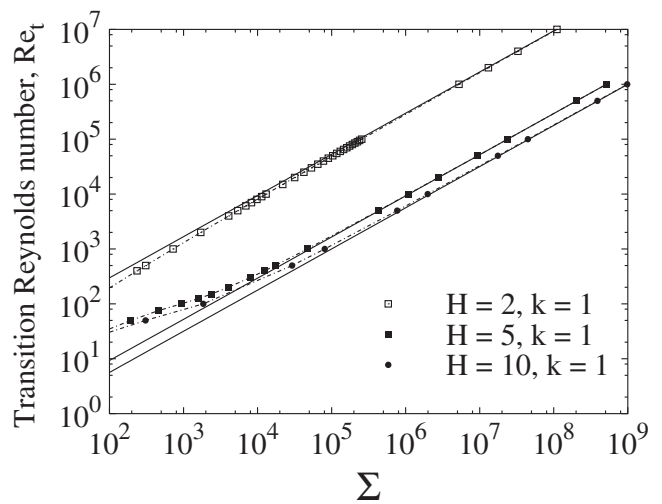

Figure 7. Neutral stability curves in the $R e-\Sigma$ plane for stability of fully developed flow to axisymmetric disturbances. Results (from Shankar \& Kumaran (2001a)) shown for different parameters indicated in the figure, and for $\eta_{r}=0$. The dotted lines show predictions from the asymptotic analysis, and the continuous lines show results from numerical solutions.

\subsection{Role of neo-Hookean solid model}

While the predictions for wall modes were derived Shankar \& Kumaran (2001a, 2002) using the linear elastic solid model, it has been found (Gaurav \& Shankar 2009) that wall modes are present in the high- Re regime even for a neo-Hookean solid. It turns out that the predictions (Gaurav \& Shankar 2009; Chokshi 2007) for most unstable wall mode remains unaffected by the use of the a neo-Hookean solid, although there are some differences in predictions for the other (less) unstable modes. Thus, for wall modes, it has been explicitly verified that the use of neo-Hookean model does not change the predictions obtained from the linear elastic solid model in the high $R e$ limit. The earlier predictions for the inviscid modes (Shankar \& Kumaran 1999, 2000) obtained using linear elastic model are similarly expected to remain unaffected with the use of a neo-Hookean solid. This is because for inviscid modes, $\rho V^{2} \sim G$, while the balance in the base state is always between $V \eta / R \sim G$. By comparing the two, it can be concluded that $V \eta /(G R) \propto R e^{-1}$ for inviscid modes. At high $R e$, the base-state strain $(\sim V \eta /(G R))$ in the solid is therefore negligible, and the predictions obtained by using the linear viscoelastic model for inviscid modes would remain unaffected.

\section{Low and intermediate Reynolds number}

In the limit of very low $R e$, the inertial stresses in the fluid are negligible compared to viscous stresses. If the deformable solid is to have any effect on the flow, then the viscous stresses in the fluid must be of the same order as the elastic stresses in the solid. If the densities of fluid and deformable solid are comparable, this would mean that the inertial stresses in the solid are negligible compared to elastic stresses except in the case of high-frequency perturbations. Kumaran et al (1994) first considered the case of planar Couette flow past a linear elastic solid of finite thickness bonded to a rigid plate at the other surface. Since inertia is neglected in the fluid and the solid, there is no time-dependence in the governing equations. The time-dependence enters through the velocity continuity conditions, and there is an additional coupling between the base flow and fluctuations in the tangential velocity condition due to the linearization of the continuity condition about the unperturbed interface. This analysis predicted that the planar 
Couette flow could become unstable even in the creeping-flow limit if $\Gamma=V \eta /(G R)$ exceeds a critical value, which is a function of the ratio of solid to fluid thickness. A similar instability was predicted in the creeping-flow limit for flow in a deformable tube by Kumaran (1995). Much later, Gkanis \& Kumar (2003) pointed out that when the flow is predicted to be unstable, the nondimensional strain in the base state $\Gamma$ is an $O(1)$ quantity, and hence the use of linearized elastic model for the solid (which assumes that the strain in the solid is $\ll 1$ ) becomes inconsistent. They suggested the use of the frame-invariant neo-Hookean model to address this limitation. A neo-Hookean solid exhibits a non-zero first normal stress difference under simple shear, and a jump in this quantity across the fluid-solid interface leads to a short-wave instability much akin to the ones studied for viscoelastic fluids (Renardy 1988). The results of Gkanis \& Kumar (2003) show that when the ratio of solid to fluid thickness is greater than 1, the results of the neoHookean model agree well with the linear elastic model. However for smaller values of solid to fluid thickness, the short-wave instability becomes the most unstable mode, and the predictions are vastly different from that of the linear elastic solid. Experiments conducted by Kumaran \& Muralikrishnan (2000); Muralikrishnan \& Kumaran (2002); Eggert \& Kumar (2004) generally agree with the predictions of the linear viscoelastic model, presumably because of the high values of ratio of solid to fluid thickness used in the experiments.

Recently, Gaurav \& Shankar (2009) studied the stability of pressure-driven flow in a neoHookean tube, and showed that in this case, the instability in the creeping-flow limit (Kumaran 1995) is absent due to the additional couplings in the neo-Hookean solid model. Thus, while the instabilities at higher Reynolds number are relatively insensitive to the nature of the constitutive model, the instability at creeping-flow appears to be sensitive to the model used. Instead, Gaurav $\&$ Shankar (2009) find a class of unstable modes in the limit of zero $\operatorname{Re}$ and $\Gamma \sim O(1)$ which involve the inertial stresses in the solid. When these unstable modes are continued numerically to higher $R e$, they merge with one of the wall modes at high $R e$. The stability of pressure-driven flow in a deformable (rectangular) neo-Hookean channel was analyzed by Gaurav \& Shankar (2009) by considering both sinuous and varicose modes. They showed that either of the two modes could be the most critical ones depending on the parameter regime, thus showing the importance of considering the modes of both symmetry. They also showed that the TS mode in a rigid channel could be stabilized or destabilized by wall deformability depending on the parameters involved such as wall thickness. This result is at variance with traditional wisdom (achieved by using simple spring-backed membranes as models for the compliant wall), and further illustrates the importance of using a realistic constitutive model for the deformable wall medium.

\section{Weakly nonlinear stability}

In order to address the phenomena that happen in the immediate vicinity of the linear instability, a weakly nonlinear analysis (Stuart 1960) is normally carried out. The goal of such an analysis is to derive a Landau equation from the governing equations of fluid flow and solid deformation.

$$
\frac{1}{A} \frac{\mathrm{d} A}{\mathrm{~d} t}=\operatorname{Re}\left(s_{0}\right)+s_{1}|A|^{2}
$$

Here, $A$ is the amplitude of the most unstable mode. This is usually achieved by employing a multiple-scales approach. If the flow is unstable, and if $\operatorname{Re}\left[s_{1}\right]>0$ the growth can become saturated as $\Gamma$ increases beyond $\Gamma_{c}$. This situation is called as supercritical bifurcation. Conversely, if the flow is stable, and if $\operatorname{Re}\left[s_{1}\right]<0$, then the flow is subcritically unstable, since a sufficiently 
large perturbation below $\Gamma_{c}$ can make the flow unstable, even if it is linearly stable. Shankar \& Kumaran (2001b) first carried out a weakly nonlinear analysis in the creeping-flow limit for a purely elastic solid, and showed that the instability is subcritical for a wide range of parameters. Choskshi \& Kumaran (2008) extended this for a neo-Hookean surface (with dissipation) and showed that the nature of the bifurcation depends critically on the ratio of gel to fluid thickness, as well as viscosity ratio (gel to fluid). The prediction that the instability is subcritical is in qualitative agreement with the experimental observations of Eggert \& Kumar (2004). Later, Chokshi (2007) carried out a weakly nonlinear analysis for wall modes as well, and showed that the instability is always supercritical at higher $R e$. Although experimental studies in flow through deformable tubes exhibit an instability (Verma \& Kumaran 2012), it is not yet clear whether there is evidence on the nature of the bifurcation.

\section{Summary}

In summary, the understanding of linear stability of internal flow through tubes and channels is now fairly mature. Broadly, three classes of instabilities are present in flow past deformable solid surfaces: at high $R e$, there is the "inviscid mode" which requires the critical layer to be present in the flow, and this is much similar to the so-called "travelling wave flutter" mode studied earlier in boundary layer flows. For inviscid modes, the transition $R e$ scales as $R e \sim \Sigma^{1 / 2}$. In addition, there is a new type of mode called the "wall mode", which was not predicted by earlier studies primarily because of the neglect of tangential wall motion. The transition $R e$ for wall modes scale as $\operatorname{Re} \sim \Sigma^{3 / 4}$. The "static divergence" mode analyzed in the earlier literature requires wall damping to be dominant at high $R e$, and this is practically impossible for continuum viscoelastic walls. In addition, there is a "viscous mode" at very low $R e$. The predictions for low $R e$ seem to be quite sensitive to the nature of the constitutive models used, while at high $R e$, predictions are relatively insensitive to the models. Experimental observations (Verma \& Kumaran 2012, 2013) in deformable circular tubes and rectangular channels do show instabilities at $R e \sim 500$, but they also show that there is a change in the tube cross-section due to applied pressure gradient. If this change is accounted for, then there is reasonably good agreement between the wall mode predictions and experimental observations, as argued by Verma \& Kumaran (2013). The question still remains as to why the inviscid mode, which is theoretically predicted to be much more unstable than the wall mode, is not observed in the experimental studies. This must be addressed in future experimental and theoretical studies. Furthermore, more realistic constitutive models could be used and their role on the predictions of instability could be investigated in the future. While the onset of the instability has been reasonably understood, not much is known about the events that occur after the instability. A combined experimental and computational approach is necessary to gain some understanding on this more challenging problem.

\section{Acknowledgements}

The author would like to thank Professor V. Kumaran, Dr. Rochish Thaokar and Dr. Paresh Chokshi for many useful discussions and suggestions, and his collaborator Dr. Gaurav with whom some of the reported work was carried out. 


\section{References}

Beatty M F and Zhou Z 1991 Universal motion for a class of viscoelastic materials of differential type. Continuum Mech. Thermodyn. 3: 169

Benjamin T B 1960 Effect of a flexible surface on boundary layer stability. J. Fluid Mech. 9: 513-532

Benjamin B 1963 The threefold classification for unstable disturbances in flexible surfaces bounding inviscid flows. J. Fluid Mech. 16: 436-450

Carpenter P W and Gajjar J S B 1990 A general theory for two and three dimensional wall-mode instabilities in boundary layers over isotropic and anisotropic compliant walls. Theoret. Comput. Fluid Dyn. 1: 349378

Carpenter P and Morris P 1990 The effect of anisotropic wall compliance on boundary-layer stability and transition. J. Fluid Mech. 218: 171-223

Carpenter P W and Garrad A D 1985 The hydrodynamic stability of flows over Kramer-type compliant surfaces. part 1. Tollmien-schlichting instabilities. J. Fluid Mech. 155: 465-510

Carpenter P W and Garrad A D 1986 The hydrodynamic stability of flows over Kramer-type compliant surfaces. part 2. flow induced surface instabilities. J. Fluid Mech. 170: 199-232

Chandrasekhar S 1981 Hydrodynamic and hydromagnetic stability. New York: Dover

Chen K P 1991 Elastic instability of the interface in Couette flow of two viscoelastic liquids. J. NonNewtonian Fluid Mech. 40: 261-267

Chokshi P 2007 Studies in the stability of newtonian and viscoelastic flows past rigid and flexible surfaces. $\mathrm{PhD}$ thesis, Indian Institute of Science, Bangalore, India

Choskshi P and Kumaran V 2007 Stability of the flow of a viscoelastic fluid past a deformable surface in the low reynolds number limit. Phys. Fluids 19: 104,103

Choskshi P and Kumaran V 2008 Weakly nonlinear analysis of viscous instability in flow past a neohookean surface. Phys. Rev. E 77: 056,303

Davies C 2003 Convective and absolute instabilities of flows over compliant walls. In: Carpenter P W and Pedley T J (eds) IUTAM symposium on flow past highly compliant boundaries and in collapsible tubes, Kluwer Academic, The Netherlands, chap 4, pp 69-94

Davies C and Carpenter P W 1997 Instabilities in a plane channel flow between compliant walls. J. Fluid Mech. 352: 205-243

Destarde M and Saccomandi G 2004 Finite-amplitude inhomogeneous waves in mooney-rivlin viscoelastic solids. Wave Motion 40: 251-262

Drazin P and Reid W 1981 Hydrodynamic stability. Cambridge: Cambridge University Press

Eggert M D and Kumar S 2004 Observations of instability, hysterisis, and oscillation in low-reynolds number flow past polymer gels. J. Colloid Interface Sci. 278: 234-242

Fosdick R L and Yu J H 1996 Thermodynamics, stability and non-linear oscillations of viscoelastic solids - i. differential type solids of second grade. Int. J. Non-Linear Mech. 31: 495

Gajjar J S B and Sibanda S K 1996 The hydrodynamic stability of channel flow with compliant boundaries. Theor. Comput. Fluid Dyn. 8: 105-129

Gaurav and Shankar V 2009 Stability of fluid flow through deformable neo-hookean tubes. J. Fluid Mech. 627: 291-322

Gkanis V and Kumar S 2003 Instability of creeping couette flow past a neo-hookean solid. Phys. Fluids 15: 2864-2471

Grotberg J B 2011 Respiratory fluid mechanics. Phy. Fluids 23(2): 021301

Grotberg J B and Jensen O E 2004 Biofluid mechanics in flexible tubes. Ann. Rev. Fluid Mech. 36: 121-147

Heil M and Jensen O E 2003 Flow in deformable tubes and channels. In: Carpenter P W, Pedley T J (eds) IUTAM symposium on flow past highly compliant boundaries and in collapsible tubes, Kluwer Academic, The Netherlands, chap 2, pp 15-49

Holzapfel G A 2000 Nonlinear solid mechanics. Chichester, UK: John Wiley

Krindel P and Silberberg A 1979 Flow through gel-walled tubes. J. Colloid Interface Sci. 71: 34-50

Ku D N 1997 Blood flow in arteries. Annu. Rev. Fluid Mech. 29: 399-434 
Kumar A S and Shankar V 2005 Instability of high-frequency modes in viscoelastic plane couette flow past a deformable wall at low and finite reynolds number. J. Non-Newtonian Fluid Mech. 125: 121-141

Kumaran V 1995 Stability of the viscous flow of a fluid through a flexible tube. J. Fluid Mech. 294: 259-281 Kumaran V 1996 Stability of an inviscid flow through a flexible tube. J. Fluid Mech. 320: 1-17

Kumaran V 1998a Stability of fluid flow through a flexible tube at intermediate Reynolds number. J. Fluid Mech. 357: 123-140

Kumaran V 1998b Stability of wall modes in a flexible tube. J. Fluid Mech. 362: 1-15

Kumaran V 2003 Hydrodynamic stability of flow through compliant channels and tubes. In: Carpenter P W and Pedley T J (eds) IUTAM symposium on flow past highly compliant boundaries and in collapsible tubes. Kluwer Academic, The Netherlands, chap 5, pp 95-118

Kumaran V and Muralikrishnan R 2000 Spontaneous growth of fluctuations in the viscous flow of a fluid past a soft interface. Phys. Rev. Lett. 84: 3310-3313

Kumaran V, Fredrickson G H and Pincus P 1994 Flow induced instability of the interface between a fluid and a gel at low Reynolds number. J. Phys. II France 4: 893-904

Lahav J, Eliezer N and Silberberg A 1973 Gel - walled cylindrical channels as models for micro-circulation: Dynamics of flow. Biorheology 10: 595-604

Landahl M T 1962 On the stability of a laminar incompressible boundary layer over a flexible surface. J. Fluid Mech. 13: 609

Landau L and Lifshitz E 1989 Theory of elasticity. New York: Pergamon

LaRose P G and Grotberg J B 1997 Flutter and long-wave instabilities in compliant channels conveying developing flows. J. Fluid Mech. 331: 37

Lucey A D and Carpenter P W 1992 A numerical simulation of the interaction of a compliant wall and inviscid flow. J. Fluid Mech. 234: 121-146

Lucey A D and Carpenter P W 1993 On the difference between the hydroelastic instability of infinite and very long compliant panels. J. Sound Vibr. 163: 176-181

Lucey A D and Peake N 2003 Wave excitation on flexible walls in the presence of a fluid flow. In: Carpenter P W and Pedley T J (eds) IUTAM symposium on flow past highly compliant boundaries and in collapsible tubes, Kluwer Academic, The Netherlands, chap 6, pp 119-146

Ma Y and Ng C O 2009 Wave propagation and induced steady streaming in viscous fluid contained in a prestressed viscoelastic tube. Phys. Fluids 21: 051,901

Macosko C 1994 Rheology: Principles, measurements and applications. New York: VCH

Malvern L E 1969 Introduction to the mechanics of a continuous medium. Englewood Cliffs, NJ: PrenticeHall

McDonald J C and Whitesides G M 2002 Poly(dimethylsiloxane) as a material for fabricating microfluidic devices. Acc. Chem. Res. 35: 491-499

Muralikrishnan R and Kumaran V 2002 Experimental study of the instability of viscous flow past a flexible surface. Phys. Fluids 14: 775-780

Neelamegam R, Shankar V and Das D 2013 Suppression of purely elastic instabilities in the torsional flow of viscoelastic fluid past a soft solid. Phys. Fluids 25: 124,102

Pedley T J 2000 Blood flow in arteries and veins. In: Batchelor G K, Moffat H K and Worster M G (eds) Perspectives in fluid dynamics, Cambridge, chap 3, pp 105-153

Renardy Y 1988 Stability of the interface in two-layer Couette flow of upper convected Maxwell liquids. J. Non-Newtonian Fluid Mech. 28: 99-115

Schmid P 2007 Nonmodal stability theory. Ann. Rev. Fluid Mech. 39: 129-162

Schmid P J and Henningson D S 2001 Stability and transition in shear flows. New York: Springer

Sen P K and Arora S K 1987 On the stability of a laminar incompressible boundary layer over a flexible surface. J. Fluid Mech. 13: 609

Shankar V and Kumar S 2004 Instability of viscoelastic plane Couette flow past a deformable wall. J. Non-Newtonian Fluid Mech. 116: 371-393

Shankar V and Kumaran V 1999 Stability of non-parabolic flow in a flexible tube. J. Fluid Mech. 395: $211-236$ 
Shankar V and Kumaran V 2000 Stability of fluid flow in a flexible tube to non-axisymmetric disturbances. J. Fluid Mech. 408: 291-314

Shankar V and Kumaran V 2001a Asymptotic analysis of wall modes in a flexible tube revisited. Eur. Phys. J. B 19: 607-622

Shankar V and Kumaran V 2001b Weakly nonlinear stability of viscous flow past a flexible surface. J. Fluid Mech. 434: 337-354

Shankar V and Kumaran V 2002 Stability of wall modes in fluid flow past a flexible surface. Phys. Fluids 14: $2324-2338$

Squires T M and Quake S R 2005 Microfluidics: Fluid physics at the nanoliter scale. Rev. Mod. Phys. 77: 977-1026

Stuart J T 1960 On the non-linear mechanics of wave disturbances in stable and unstable parallel flows: Part 1. the basic behaviour in plane poiseuille flow. J. Fluid Mech. 9: 353-370

Verma M K S and Kumaran V 2012 A dynamical instability due to fluid-wall coupling lowers the transition reynolds number in the flow trough a flexible tube. J. Fluid Mech. 705: 322-347

Verma M K S and Kumaran V 2013 A multifold reduction in the transition reynolds number, and ultra-fast mixing, in a micro-channel due to a dynamical instability induced by a soft wall. J. Fluid Mech. 727: 407-45

Yeo K 1988 The stability of boundary layer flow over single and multilayer viscoelastic walls. J. Fluid Mech. 196: 359

Yeo K and Dowling A 1987 The stability of inviscid flows over passive compliant walls. J. Fluid Mech. 183: 265-292

Yeo K, Khoo B and Chong W 1994 The linear stability of boundary layer flow over compliant walls: The effects of wall mean state induced by flow loading. J. Fluids Struct. 8: 529 